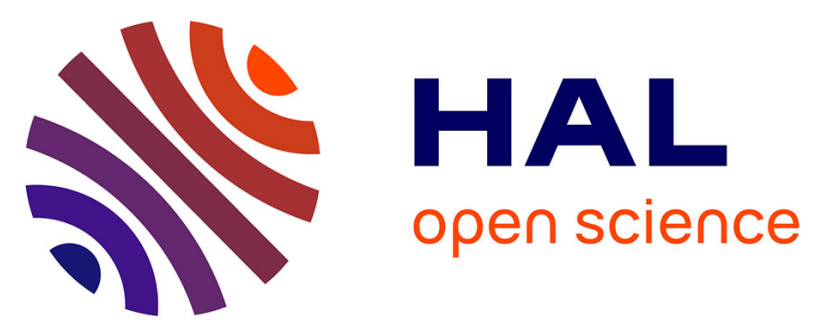

\title{
Rapid Adoption of Telemedicine in Rheumatology Care During the COVID -19 Pandemic Highlights Training and Supervision Concerns Among Rheumatology Trainees
}

Su-ann Yeoh, Kristen Young, Michael Putman, Sebastian Sattui, Richard Conway, Elizabeth Graef, Adam Kilian, Maximilian Konig, Jeffrey Sparks, Manuel Ugarte-gil, et al.

\section{- To cite this version:}

Su-ann Yeoh, Kristen Young, Michael Putman, Sebastian Sattui, Richard Conway, et al.. Rapid Adoption of Telemedicine in Rheumatology Care During the COVID -19 Pandemic Highlights Training and Supervision Concerns Among Rheumatology Trainees. ACR Open Rheumatology, 2021, 10.1002/acr2.11355 . hal-03451504

\section{HAL Id: hal-03451504 https://hal.sorbonne-universite.fr/hal-03451504}

Submitted on 26 Nov 2021

HAL is a multi-disciplinary open access archive for the deposit and dissemination of scientific research documents, whether they are published or not. The documents may come from teaching and research institutions in France or abroad, or from public or private research centers.
L'archive ouverte pluridisciplinaire HAL, est destinée au dépôt et à la diffusion de documents scientifiques de niveau recherche, publiés ou non, émanant des établissements d'enseignement et de recherche français ou étrangers, des laboratoires publics ou privés. 


\title{
Rapid Adoption of Telemedicine in Rheumatology Care During the COVID-19 Pandemic Highlights Training and Supervision Concerns Among Rheumatology Trainees
}

\author{
Su-Ann Yeoh, ${ }^{1}$ (D) Kristen Young, ${ }^{2}$ (D) Michael Putman, ${ }^{3}$ (D) Sebastian Sattui, ${ }^{4}$ (D) Richard Conway, ${ }^{5}$ (DD \\ Elizabeth Graef, ${ }^{6}$ (D) Adam Kilian, ${ }^{7}$ Maximilian Konig, ${ }^{8}$ iD Jeffrey Sparks, ${ }^{9}$ iD Manuel Ugarte-Gil, ${ }^{10}$ (DD \\ Laura Upton, ${ }^{11}$ Francis Berenbaum, ${ }^{12}$ Suleman Bhana, ${ }^{13}$ Wendy Costello, ${ }^{14}$ Jonathan Hausmann, ${ }^{15}$ \\ Pedro Machado, ${ }^{1}$ (D) Philip Robinson, ${ }^{16}$ Emily Sirotich, ${ }^{17}$ Paul Sufka, ${ }^{18}$ Jinoos Yazdany, ${ }^{19}$ (D) Jean Liew, ${ }^{20}$ \\ Rebecca Grainger, ${ }^{21}$ (D) Zachary Wallace, ${ }^{22}$ and Arundathi Jayatilleke, ${ }^{23}$ The Global Rheumatology \\ Alliance
}

Objective. To evaluate the impact of telemedicine use during the coronavirus disease 2019 (COVID-19) pandemic on rheumatology trainees.

Methods. A voluntary, anonymous, web-based survey was administered in English, Spanish, or French from August 19 to October 5, 2020. Adult and pediatric rheumatology trainees were invited to participate via social media and email. Using multiple-choice questions and Likert scales, the survey assessed prior and current telemedicine use, impact on training, and supervision after COVID-19 prompted rapid telemedicine implementation.

Results. Surveys were received from 302 trainees from 33 countries, with $83 \%$ in adult rheumatology training programs. Reported telemedicine use increased from $13 \%$ before the pandemic to $82 \%$ during the pandemic. United States trainees predominantly used video visits, whereas outside the United States telemedicine was predominantly audio only. Most (65\%) evaluated new patients using telemedicine. More respondents were comfortable using telemedicine for follow-up patients $(69 \%)$ than for new patients $(25 \%)$. Only $39 \%$ of respondents reported receiving telemedicine-focused training, including instruction on software, clinical skills, and billing, whereas more than half of United States trainees $(59 \%)$ had training. Postconsultation verbal discussion was the most frequent form of supervision; $24 \%$ reported no supervision. Trainees found that telemedicine negatively impacted supervision (50\%) and the quality of clinical teaching received $(70 \%)$, with only $9 \%$ reporting a positive impact.

Conclusions. Despite widespread uptake of telemedicine, a low proportion of trainees received telemedicine training, and many lacked comfort in evaluating patients, particularly new patients. Inadequate supervision and clinical teaching were areas of concern. If telemedicine remains in widespread use, ensuring appropriate trainee supervision and teaching should be prioritized.

No funding was received for this study.

The views expressed here are those of the authors and participating members of the COVID-19 Global Rheumatology Alliance and do not necessarily represent the views of the American College of Rheumatology, the European League Against Rheumatism, or any other organization.

${ }^{1}$ Su-Ann Yeoh, MRCP, BMBS, BmedSci, Pedro Machado, FRCP, PhD, University College London, United Kingdom; ${ }^{2}$ Kristen Young, DO, Division of Rheumatology, University of Arizona College of Medicine, Phoenix; ${ }^{3}$ Michael Putman, MD, Msci, Medical College of Wisconsin, Milwaukee; ${ }^{4}$ Sebastian Sattui, MD, MS, Division of Rheumatology and Clinical Immunology, University of Pittsburgh, Pittsburgh, PA; ${ }^{5}$ Richard Conway, MBBChBAO, PhD, St James's Hospital, Dublin, Ireland; ${ }^{6}$ Elizabeth Graef, DO, Emerson Hospital, Concord, Massachusetts; ${ }^{7}$ Adam Kilian, MD, The George Washington University, Washington, DC; ${ }^{8}$ Maximilian Konig, MS, Johns Hopkins University, Baltimore, Maryland; ${ }^{9}$ Jeffrey Sparks, MD, MMSc, Brigham and Women's Hospital, Boston, Massachusetts; ${ }^{10}$ Manuel Ugarte-Gil, MD, MSc, Universidad Cientifica del Sur, Lima, Peru; ${ }^{11}$ Laura Upton, BA,
Georgetown University, Washington, DC; ${ }^{12}$ Francis Berenbaum, MD, PhD, Hopital Saint-Antoine, Paris, France; ${ }^{13}$ Suleman Bhana, MD, FACR, Crystal Run Health, Middletown, New York; ${ }^{14}$ Wendy Costello, Irish Children's Arthritis Network, Tipperary, Ireland; ${ }^{15}$ Jonathan Hausmann, MD, Boston Children's Hospital, Massachusetts; ${ }^{16}$ Philip Robinson, MBDhB, PhD, FRACP, MAICD, University of Queensland, Brisbane, Queensland, Australia; ${ }^{17} \mathrm{Emily}$ Sirotich, BSc, McMaster University, Canadian Arthritis Patient Alliance, Hamilton, Ontario, Canada; ${ }^{18}$ Paul Sufka, MD, HealthPartners, St. Paul, Minnesota; ${ }^{19}$ Jinoos Yazdany, MD, MPH, University of California in San Francisco; ${ }^{20}$ Jean Liew, MD, MS, Boston University, Massachusetts; ${ }^{21}$ Rebecca Grainger, MBChB, BmedSci, PhD, University of Otago, Wellington, New Zealand; ${ }^{22}$ Zachary Wallace, MD, MSc, Massachusetts General Hospital, Boston; ${ }^{23}$ Arundathi Jayatilleke, MD, MS, Temple University Philadelphia, Pennsylvania

Drs. Yeoh and Young contributed equally to this work.

Dr. Yeoh has received research grants from the Royal College of Physicians, Rosetrees Trust, National Institute of Health Research University 


\section{INTRODUCTION}

The novel coronavirus disease 2019 (COVID-19) pandemic demanded a rapid, unplanned change in the mode of delivery of health care services. Providers were required to balance the dual necessities of reducing nonemergent in-person clinical contact while maintaining patient access to care. The majority of rheumatology care occurs in the outpatient setting, which saw a rapid increase in the use of telephone or video calling to deliver care remotely, also known as telemedicine (1). This has implications not only for patient care but also for rheumatology training. There is a paucity of data regarding rheumatology trainee experiences with telemedicine during the pandemic. The COVID-19 Global Rheumatology Alliance (GRA), an international collaborative of the rheumatology community, was formed to collect data in rheumatology relevant to COVID-19 (2). The objective of this study was to assess the use of telemedicine before and during the COVID-19 pandemic and the impact of telemedicine on rheumatology trainees' experiences during the COVID-19 pandemic.

\section{METHODS}

We developed multiple-choice and Likert-based survey questions pertaining to telemedicine (15 in total; see Supplementary Material) as part of a 77-question trainee survey for the COVID-19 GRA. The survey was piloted with five rheumatology trainees (from the United States and the United Kingdom; two were pediatric rheumatology trainees) and revised according to feedback. The survey was available in English, Spanish, and French via the REDCap (Project R) platform to adult and pediatric rheumatology trainees. Data from the United Kingdom/European Economic Area (EEA) were hosted on a REDCap database at University College London in the United Kingdom, and non-United Kingdom/EEA data were hosted at Temple University Hospital in the United States. All members of the GRA, American College of Rheumatology (ACR) fellows-in-training listserv, and the Emerging European League Against Rheumatism (EULAR) Network

College London Hospitals Biomedical Research Centre, and University College London Hospitals Charities. Dr. Conway has received consulting fees, speaking fees, and honoraria from Janssen, Roche, Sanofi, and Abbvie (all less than $\$ 10$ 000). Mr. Konig has received consulting fees, speaking fees, and honoraria from Celltrion (less than $\$ 10$ 000). Dr. Sparks has received consulting fees from Bristol-Myers Squibb, Gilead, Inova Diagnostics, Optum, and Pfizer (less than \$10 000). Dr. Ugarte-Gil has received research grants from Pfizer and Janssen. Dr. Suleman Bhana has done nonbranded consulting work for Abbvie, Horizon, Novartis, and Pfizer (all less than \$10 000). Dr. Jonathan Hausmann has received consulting fees, speaking fees, and honoraria from Novartis, Biogen, and Pfizer (less than \$10 000). Dr. Machado has received consulting fees, speaking fees, and honoraria from Abbvie, bristol myers squibb, Celgene, Eli Lilly, Galapagos, Janssen, Merck pharmaceutical, Novartis, Orphazyme, Pfizer, Roche, and union chimique belge (UCB), (all less than $\$ 10$ 000). Dr. Robinson has received consulting fees, speaking fees, and honoraria from Abbvie, Atom Biosciences, Janssen, Lilly, Novartis, Pfizer, Roche, and UCB (less than \$10 000); he has also received research grants from Janssen, Novartis, Pfizer, and UCB. MS. Sirotich has received nonfinancial support from the Canadian Arthritis Patient Alliance; she is also a board
(EMEUNET) trainee listserv were contacted by email and invited to participate. A request to share the survey among their membership was made to the International League of Associations for Rheumatology Executive Committee, comprising the leadership from the ACR, African League of Associations for Rheumatology, Asia Pacific League of Associations for Rheumatology, EULAR, and Pan-American League of Associations for Rheumatology. Regional/country representatives from the GRA were also asked to disseminate invitations to rheumatology trainees from their country. United States fellowship program directors were encouraged to send invitations to their fellows. Additionally, respondents were recruited on social media using invitations from official Twitter accounts of the GRA, ACR, and EMEUNET. All surveys were completed between August 19, 2020, and October 5, 2020.

Respondents were included if they were physicians who reported, 1) rheumatology training as part of their job description, 2) were aged 18 or more, and 3) consented to participate in the survey. Respondents were excluded if they completed rheumatology training prior to 2020. The survey was voluntary and did not include personal identifiers, protected health information, or incentives for participation. To allow for the variation in the duration of rheumatology training across regions, we included eight options for the training stage (from training Years 1 to 7 or beyond and whether respondents had completed their training in 2020).

Ethics statement. Ethical approval was granted by the Institutional Review Board at Temple University Hospital (protocol 27279), by the National Research Ethics Committee for COVID19, Ireland (application number 20-NREC-COV-073), and University College London Research Ethics Committee (ethics approval identification 18859/001).

Reporting and statistical analysis. Data are reported as descriptive statistics and presented as percentages followed by the number. Data from five-point Likert scales (with anchors strongly disagree to strongly agree, very negatively impact to very

member of the COVID-19 Global Rheumatology Alliance and has received honoraria from the COVID-19 Global Rheumatology Alliance. (all less than $\$ 10000)$. Dr. Yazdany has received consulting fees, speaking fees, and honoraria from Pfizer, Astra Zeneca, Eli Lilly, and Aurinia (less than \$10 000). Dr. Liew has received research grant funding from Pfizer (more than $\$ 10000)$. Dr. Grainger has received honoraria for speaking from Pfizer Australia, Cornerstones, Jannsen New Zealand, and Jannsen Australia; and has received consultancy fees from Novartis (all less than $\$ 10000$ ). No other potential conflicts of interest relevant to this article were reported.

Author disclosures are available at https://onlinelibrary.wiley.com/action/ downloadSupplement?doi=10.1002\%2Facr211355\&file=acr211355-sup0002-Disclosureform.pdf.

Address for correspondence to Arundathi Jayatilleke, MD, MS, Temple University School of Medicine, 3322 N Broad St, Suite 201, Philadelphia, PA, United States of America 19140. Email: arundathi.jayatilleke@tuhs. temple.edu

Submitted for publication May 23, 2021; accepted in revised form September 8, 2021 
positively impact, or not helpful to very helpful) are also reported as the percentage followed by the number. Neutral (or no impact) components of the Likert scales are not reported, and only the positive and negative components of the Likert scales are reported, in which the extent of each component is specified (ie, agree and strongly agree). Data are presented using descriptive statistics and processed using R Studio version 1.4.1103.

\section{RESULTS}

A total of 302 respondents from 33 countries (Figure 1) completed the survey, with 116 (38\%) from the United States, 89 (29\%) from Europe, and 97 (32\%) from the rest of the world (ROW). The majority of respondents (252 [83\%]), were enrolled in single-track adult rheumatology training, 29 (10\%) were in single-track pediatric rheumatology training, and $7 \%(n=21)$ were in dual adult/pediatric training. The majority (279 [92\%]) were in full-time training before the COVID-19 pandemic. Most respondents reported being in the first 3 years of training ( $n=204,68 \%$; Year 1: $n=64$; Year 2: $n=85$; and Year $3 n=55), 60$ (20\%) were in Years 4 and above, and 38 (13\%) completed training in 2020.

Experience in telemedicine. Reported use of telemedicine increased from 39 trainees (13\%) before the pandemic to 247 trainees (82\%) during the pandemic. Differences were observed in the modalities used; United States trainees predominantly used video telemedicine compared with trainees from Europe and the ROW combined ( $P<0.001$ using Fisher's exact test), who predominantly used audio-only telemedicine compared with United States trainees $(P<0.001$ using Fisher's exact test) (Table 1).
For pediatric-only rheumatology trainees, 27 (93\%) reported using telemedicine during the pandemic, whereas, for those in combined adult/peds training, 19 (90\%) reported using telemedicine during the pandemic. Eighty percent of adult rheumatology trainees $(n=201)$ reported using telemedicine.

Clinical training in telemedicine and supervision. Just more than one-third ( $n=97$ [39\%]) of trainees reported that they had received training in telemedicine; the majority found telemedicine training somewhat or very helpful $(n=89$ [92\%]). A higher proportion of United States trainees $(n=69$ [62\%]) reported having received training compared with $135(21 \%)$ trainees from Europe and the ROW combined $(P<0.001$ using Fisher's exact test). Technology and platform use were more frequently addressed by this training $(n=85$ [88\%]) compared with clinical skills $(n=37$ [38\%]) and billing ( $\mathrm{n}=39$ [40\%]).

Supervision of telemedicine visits occurred most frequently by verbal discussion after the consultation. Half of the trainees ( $n=125$ [51\%]) reported supervision via postvisit discussions during the pandemic, which was similar to the proportion of trainees ( $n=18$ [46\%]) who had postvisit discussion supervision when using telemedicine before the pandemic (Table 1). Some trainees reported real-time observation for part ( $n=71$ [29\%]) or the entirety $(n=43[17 \%])$ of the telemedicine visit. Almost onequarter (59 [24\%]) of those who used telemedicine during the pandemic reported having no telemedicine supervision. Telemedicine was reported to negatively or slightly negatively impact the supervision of $50 \%$ of trainees ( $n=123$ ), whereas only $9 \%$ reported a slight or very positive impact on supervision $(n=21)$.

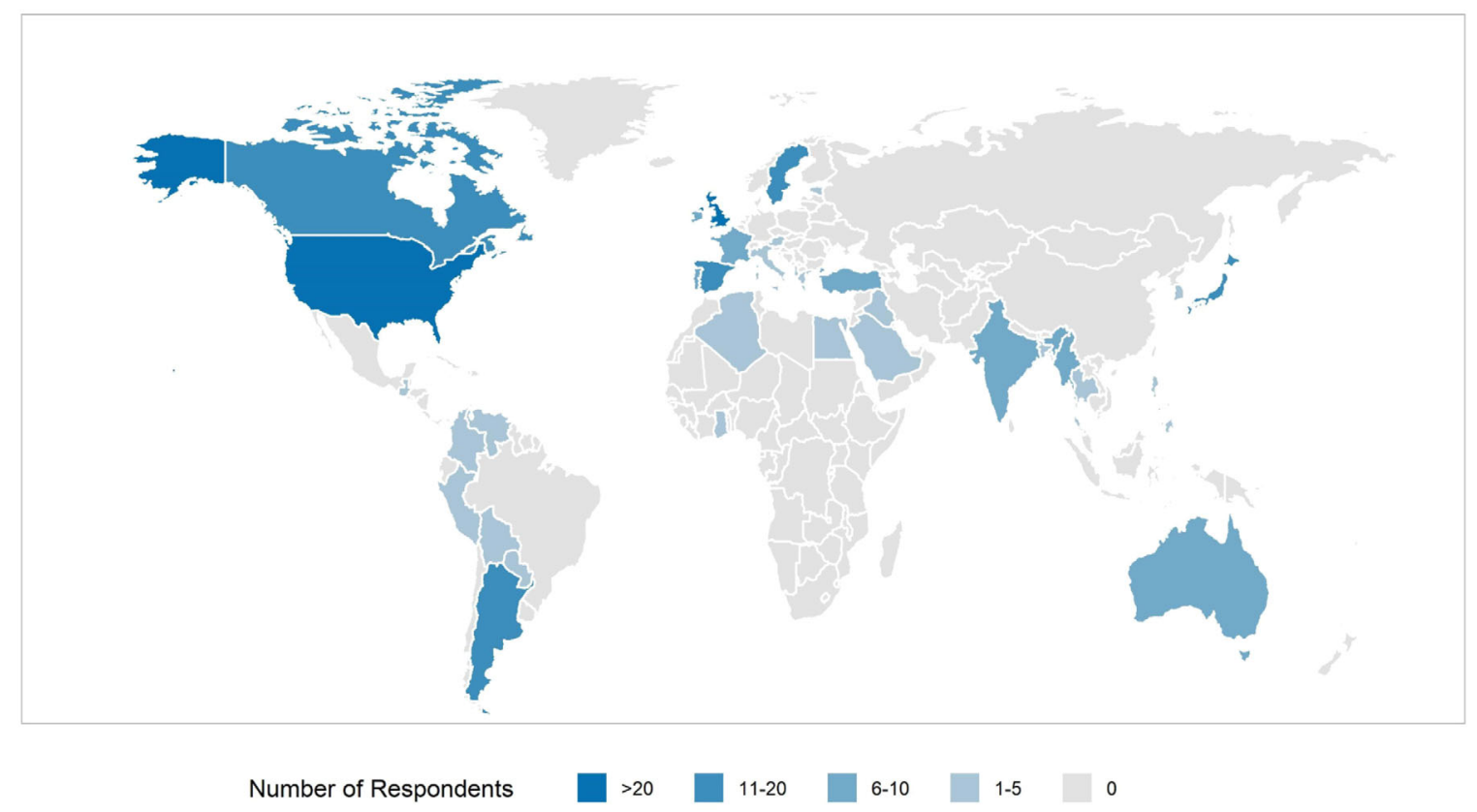

Figure 1. Map displaying the distribution of survey respondents. 
Table 1. Telemedicine use, supervision, and training by region

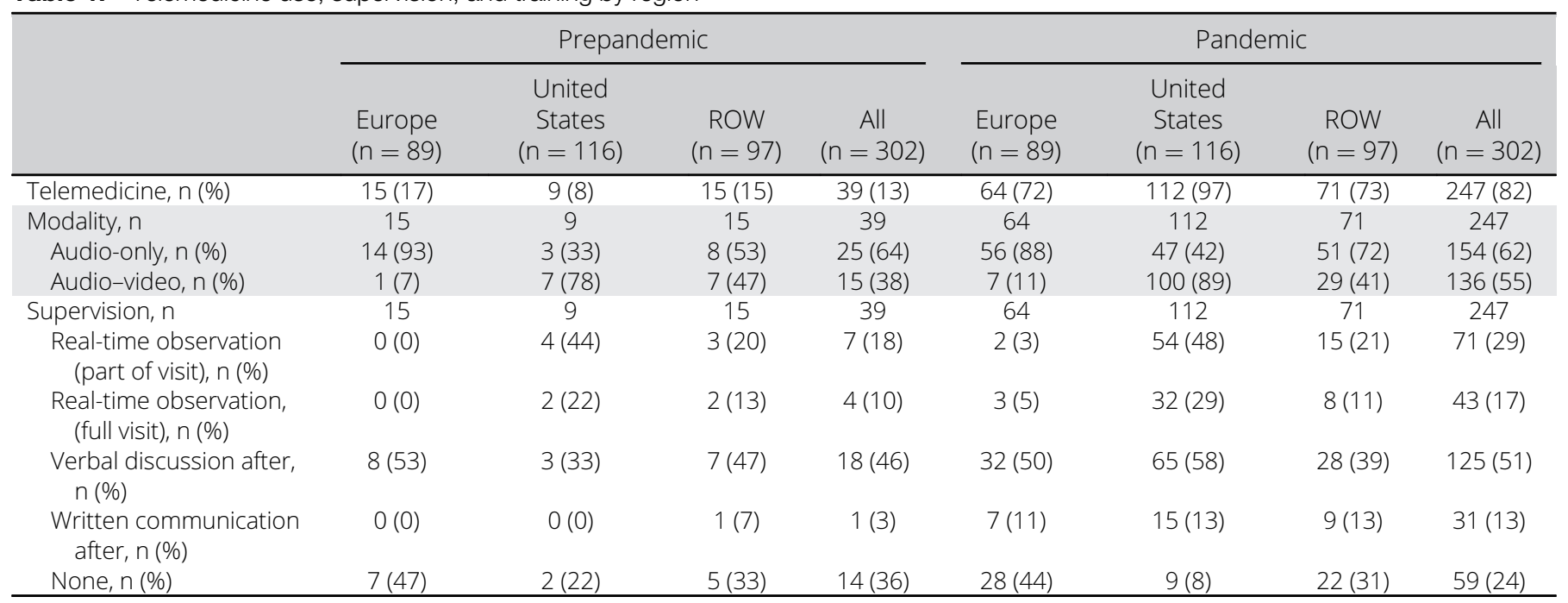

Abbreviation: ROW, rest of the world.

ROW data include Asia $(n=50)$, Central and South America $(n=23)$, Canada $(n=12)$, Australia $(n=8)$, and Africa $(n=4)$.

\section{A: Comfort level with using telemedicine during the COVID-19 Pandemic}

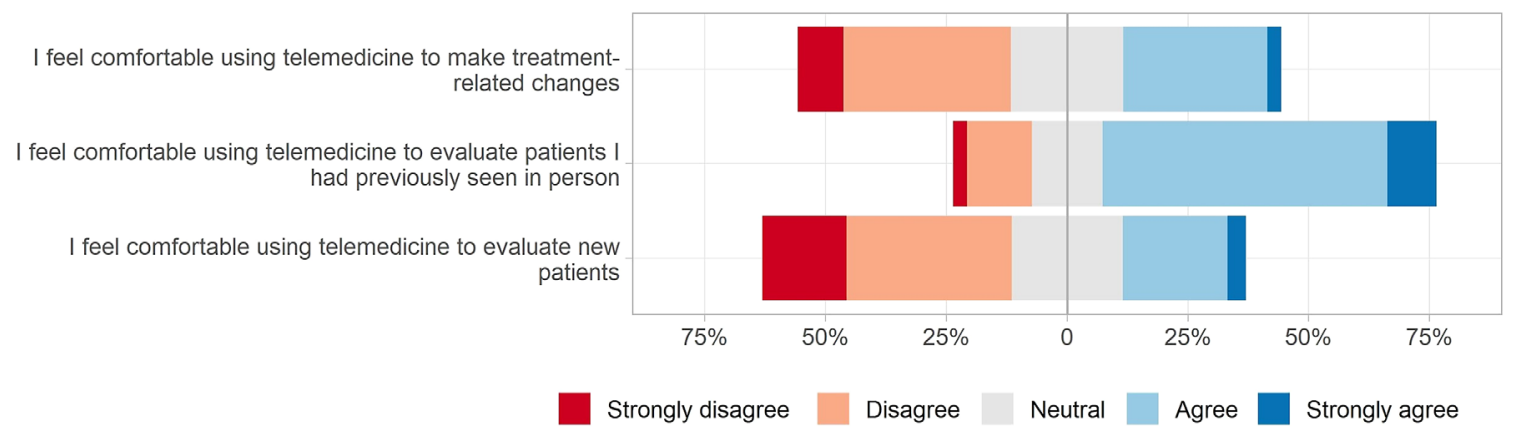

\section{B: Impact of telemedicine on trainee experience}

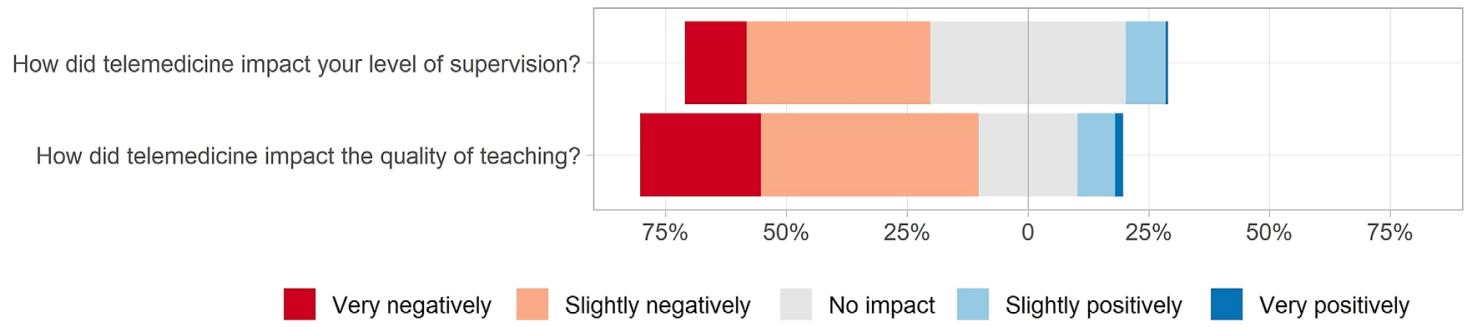

Figure 2. Rheumatology trainee comfort levels in using telemedicine during the pandemic.

For clinical teaching quality, most respondents $(\mathrm{n}=171$ [70\%]) reported that telemedicine had a negative or slightly negative impact, and only 23 (9\%) reported a slight or very positive impact on each of these areas (Figure 2).

Patients evaluated using telemedicine. Most trainees using telemedicine evaluated new patients during the pandemic $(n=161$ [65\%]). A larger proportion of trainees $(n=170$ [69\%]) agreed or strongly agreed that they felt comfortable using telemedicine to evaluate follow-up patients compared with evaluating new patients $(\mathrm{n}=41[25 \%])(P<0.01$ using Fisher's exact test) and also compared with managing changes in treatment using telemedicine $(\mathrm{n}=80$ [33\%]) $(P<0.01$ using Fisher's exact test) (Figure 2).

\section{DISCUSSION}

This large international survey of rheumatology trainees found limited prepandemic experience of telemedicine, which increased during the pandemic to $83 \%$ of respondents with a 
perceived negative impact on training. Similar increases in telemedicine use have been reported by other trainees $(3,4)$. Telemedicine training was not widespread or comprehensive, and trainees reported a negative impact of telemedicine on their clinical training and supervision. A significantly larger proportion of trainees reported feeling comfortable with evaluating follow-up patients compared with new patients or those requiring treatment changes. These findings provide insights to drive curricular design for training in and during telemedicine.

The rapid telemedicine adoption during the pandemic was focused on meeting clinical needs and was not optimized for trainee experiences, in which some trainees reported not having telemedicine-specific training and, at times, supervision. The negative impact on clinical training is likely not to be merely due to the rapid adoption of telemedicine but also due to the limited opportunities for in-person clinical encounters, crucial for training in rheumatic diseases. Standard rheumatological assessments rely on physical examination, especially during diagnosis and treatment escalation/de-escalation, which may explain why more trainees reported higher comfort levels using telemedicine for follow-up compared with new patients. In addition, only one-third of respondents were comfortable making treatment changes using telemedicine. Similarly, in a COVID-19 tele-rheumatology audit, clinicians were less likely to make a diagnosis via telemedicine, de-escalate immunosuppressive treatment, and discharge patients (5). The ideal context for trainee telemedicine experiences, then, may be in the assessment of stable follow-up patients. Providing access to rheumatology care to new patients via telemedicine must be balanced with the educational needs of trainees, wherein in-person or bedside rheumatology teaching still plays an important role and cannot fully be replaced by telemedicine-only training.

The negative impact of telemedicine on clinical teaching reported by most trainees should prompt rheumatology programs to examine preexisting curricula and ensure that telehealth competencies are incorporated such as that recommended by the Association of American Medical Colleges (6). In addition, allocating in-person visits to lessexperienced (eg, first year) trainees until they become confident (7) and ensuring that trainees possess the necessary foundational knowledge and skills before virtual visits (8) may improve the training experience.

Although telemedicine training was infrequent, it was generally helpful, though focused on technological competence. Increasing focus on telemedicine-specific clinical skills will be required, which could include essential remote physical examination skills, simulated appointments with relevant rheumatological clinical scenarios, content about legislation, and ethics relevant to managing telemedicine visits. In addition, peer mentoring or discussion groups may be useful in terms of providing a safe space to discuss difficult or challenging cases or aspects of clinical assessment that are problematic. A future telemedicine curriculum could be designed using previously identified learning needs and effective educational strategies (9).

Medical education institutions have already begun incorporating telemedicine into their curricula (10), paralleling an exponential growth in telemedicine practice. Since the pandemic, the United States Accreditation Council of Graduate Medical Education has adopted residency training requirements (Common Program Requirements) embedding telemedicine training (11). Foci will include telemedicine-specific clinical competencies in a virtual setting, virtual communication skills, and more nuanced aspects such as "webside manner" (8). Globally, telemedicine implementation will differ according to resource setting (12), community needs, and regulations (13).

Similar to our results, gastroenterology trainees reported minimal supervision during pandemic telemedicine (3). Half of the trainees indicated that telemedicine negatively impacted their supervision. There are various means by which supervision can be delivered, including real-time observation with a supervisor physically present with the trainee or through co-attendance in remote consultation; the latter has been a successful model for remote supervision of rural medical trainees (14). A successful model of remote supervision focuses on establishing a learning relationship between supervisor and junior, a stimulus for learning, and an understanding of the practicalities of remote supervision. These principles will be useful as a guide for programs and supervisors to foster a conducive learning environment for trainees (14).

Our study has limitations. Selection and response bias were difficult to minimize, but a wide recruitment strategy was used. Survey respondents were mostly United States and European trainees, limiting generalizability. We were unable to obtain information about factors that may contribute to variable training and trainee experiences, such as regional infrastructure of telemedicine, local severity of the COVID-19 pandemic, and local policy. Finally, our study was one of several surveys being distributed to trainees, which may have contributed to survey fatigue and decreased participation.

The key strength of our survey is that it is the only international rheumatology trainee survey during the pandemic of which we are aware. Our survey was designed by a multiregional team and piloted by trainees and program directors.

In conclusion, our findings emphasize the need for telemedicine to be included in the rheumatology curriculum, especially regarding teaching telemedicine-specific clinical skills, supporting trainees evaluating new or complex patients using telemedicine, and ensuring adequate supervisory arrangements.

\section{ACKNOWLEDGMENTS}

The authors would like to thank all rheumatology trainees who participated in the survey, as well as the COVID-19 Global Rheumatology Alliance for mentorship and feedback. 


\section{AUTHOR CONTRIBUTIONS}

All authors were involved in drafting the article or revising it critically for important intellectual content, and in the final approval of the version of the article to be published.

Study conception and design. Yeoh, Young, Putman, Sattui, Conway, Graef, Kilian, Sparks, Ugarte-Gil, Machado, Sufka, Liew, Grainger, Wallace, Jayatilleke.

Acquisition of data. Yeoh, Young, Ugarte-Gil, Berenbaum, Sufka, Jayatilleke.

Analysis and interpretation of data. Yeoh, Young, Putman, Sattui, Conway, Graef, Kilian, Konig, Sparks, Ugarte-Gil, Upton, Berenbaum, Bhana, Costello, Hausmann, Machado, Robinson, Sirotich, Yazdany, Liew, Grainger, Wallace, Jayatilleke.

\section{REFERENCES}

1. Gkrouzman E, Wu DD, Jethwa H, Abraham S. Telemedicine in rheumatology at the advent of the COVID-19 pandemic. HSS J 2020; 16:1-4.

2. Wallace ZS, Bhana S, Hausmann JS, Robinson PC, Sufka P, Sirotich $E$, et al. The rheumatology community responds to the COVID-19 pandemic: the establishment of the COVID-19 global rheumatology alliance. Rheumatology (Oxford) 2020;59:1204-6.

3. Keihanian T, Sharma P, Goyal J, Sussman DA, Girotra M. Telehealth utilization in gastroenterology clinics amid the covid-19 pandemic: impact on clinical practice and gastroenterology training. Gastroenterology 2020;159:1598-601.

4. Kahwash BM, Deshpande DR, Guo C, Panganiban CM, Wangberg H, Craig TJ. Allergy/immunology trainee experiences during the COVID19 pandemic: AAAAI work group report of the fellows-in-training committee. J Allergy Clin Immunol Pract 2021;9:1-6.e1.

5. Zhu W, de Silva T, Eades L, Morton S, Ayoub S, Morand E, et al. The impact of telerheumatology and COVID-19 on outcomes in a tertiary rheumatology service: a retrospective audit. Rheumatology (Oxford) 2021;60:3478-80.
6. Association of American Medical Colleges. Telehealth competencies across the learning continuum. AAMC new and emerging areas in medicine series. Washington (DC): Association of American Medical Colleges. 2021. URL: https://www.aamc.org/what-we-do/missionareas/medical-education/cbme/competency.

7. Dua AB, Kilian A, Grainger R, Fantus SA, Wallace ZS, Buttgereit F, et al. Challenges, collaboration, and innovation in rheumatology education during the COVID-19 pandemic: leveraging new ways to teach. Clin Rheumatol 2020;39:3535-41.

8. Lockwood MM, Wallwork RS, Lima K, Dua AB, Seo P, Bolster MB. Telemedicine in adult rheumatology: in practice and in training. Arthritis Care Res (Hoboken) 2021. E-pub ahead of print.

9. Stovel RG, Gabarin N, Cavalcanti RB, Abrams H. Curricular needs for training telemedicine physicians: a scoping review. Med Teach 2020; 42:1234-42.

10. Waseh S, Dicker AP. Telemedicine training in undergraduate medical education: mixed-methods review. JMIR Med Educ 2019;5:e12515.

11. ACGME Response to COVID-19: clarification regarding telemedicine and ACGME surveys. 2020. URL: https://www.acgme.org/Newsroom/Blog/ Details/ArticleID/10125/ACGME-Response-to-COVID-19-Clarificationregarding-Telemedicine-and-ACGME-Surveys.

12. World Health Organization. Telemedicine: opportunities and developments in member states: report on the second global survey on eHealth. World Health Organization. 2010. URL: https://www.who. int/goe/publications/goe_telemedicine_2010.pdf.

13. Europe Economics. Regulatory approaches to telemedicine. 2018. URL: https://www.gmc-uk.org/about/what-we-do-and-why/dataand-research/research-and-insight-archive/regulatory-approachesto-telemedicine.

14. Cameron M, Ray R, Sabesan S. Remote supervision of medical training via videoconference in northern Australia: a qualitative study of the perspectives of supervisors and trainees. BMJ Open 2015;5:e006444.

15. Devadula S, Langbecker D, Vecchio P, Tesiram J, Meiklejohn J, Benham $\mathrm{H}$. Tele-rheumatology to regional hospital outpatient clinics: patient perspectives on a new model of care. Telemed $\mathrm{J} E$ Health 2020;26:912-9. 